of post-operative OD, these results will allow speech pathologists to provide more targeted assessment, intervention and management of OD to prevent related complications. Further investigation is essential in order to fully understand predisposing factors to developing OD in this population.

\title{
Occupational tuberculosis in health care workers in sub-Saharan Africa: A systematic review
}

\begin{abstract}
Faith O. Alele, Richard C. Franklin, Peter A. Leggat, Theophilus I. Emeto
Public Health and Tropical Medicine, College of Public Health, Medical and Veterinary Sciences, James Cook University, Townsville, Queensland

Background: Tuberculosis (TB) is a leading cause of death globally. Sub-Saharan Africa (SSA) in particular has a high burden of TB, which places health care workers (HCWs) at increased risk of occupational exposure to TB. Aim: To describe the incidence, and prevalence of latent TB infection/disease in HCWs, and explore the effectiveness of infection control measures to protect HCWs in SSA. Method: A systematic review following the Preferred Reporting Items for Systematic Reviews and Meta-Analyses (PRISMA) statement was conducted using the databases: PubMed, Web of Science, Scopus, and Medline, up to March 2016. Thirteen studies were found reporting the prevalence, incidence of latent TB infection/TB disease among HCWs, and the effectiveness of infection control strategies in SSA. Results: The median prevalence of latent TB infection (using a positive tuberculin skin test) in HCWS was $61 \%$ (range $45 \%$ to $84 \%$ ). Markers of occupational exposure associated with latent TB infection among HCWS were longer duration of work, and exposure to TB patients. The median annual incidence of TB infection attributable to occupational exposure was $29 \%$ (range $19.3 \%$ to $38 \%$ ) after accounting for the infection in the general population. The rate of active TB in HCWS was higher than in the general population. Administrative, personal and engineering control measures had no impact on the development of TB disease. Conclusions: The risk of acquiring TB disease among HCWs in SSA was high and was associated with poor infection control measures. This may impact on the recruitment, longevity and retention of HCWs.
\end{abstract}

\section{Economic evaluation of tamsulosin for the treatment of ureteric stones by size and location}

\author{
Greg Merlo, Tilley Pain²,3, Leonie Jones ${ }^{4}$, Jeremy Furyk ${ }^{3,4,5,6}$ \\ ${ }^{1}$ Australian Centre for Health Services Innovation (AusHSI), Queensland University of Technology, Brisbane, Queensland \\ ${ }^{2}$ Allied Health Management Unit, The Townsville Hospital, Townsville, Queensland \\ ${ }^{3}$ College of Public Health, Medical and Veterinary Sciences, James Cook University, Townsville, Queensland \\ ${ }^{4}$ Emergency Department, The Townsville Hospital, Townsville, Queensland \\ ${ }^{5}$ Townsville Hospital and Health Service, Townsville, Queensland \\ ${ }^{6}$ School of Medicine, University of Queensland, Herston, Queensland
}

Background: Ureteric stones are a common ailment presenting to emergency departments in Australia leading to significant distress to patients and economic consequences to the healthcare system. This economic evaluation assesses the economic impact of funding tamsulosin for the treatment or ureteric stones-by size and location-in the Australian setting. Method: A decision analytic model was designed to compare the cost of tamsulosin and initial observation for the treatment of ureteric stones by stone size and location. Clinical and epidemiological literature informed the estimates of base rate of spontaneous passage by stone size and location as well as the treatment effect of tamsulosin. Costs were measured in 2016 Australian Dollars from a health sector perspective. Results: For large distal stones, tamsulosin was significantly cost saving compared with placebo in the base scenario $(\$ 763.91 ; 95 \% \mathrm{Cl} \$ 110.39$, $\$ 1,417.42)$ and the alternative scenario (\$879.18; 95\% Cl \$396.34, \$1,362.01) per patient). Tamsulosin non-significantly increased costs for small distal stones and proximal stones. Conclusions: This decision analysis recommends emergency department consultants use tamsulosin as a cost effective management strategy for patients with large $(\geq 5$ $\mathrm{mm}$ ) distal ureteric stones.

\section{A meta-analysis of the efficacy of allopurinol in reducing the incidence of myocardial infarction}

\author{
Aarya Murali, Sean A. Morrison, Joseph V. Moxon, Jonathan Golledge \\ Queensland Research Centre for Peripheral Vascular Disease (QRCPVD), James Cook University, Townsville, Queensland
}

Background: Myocardial infarction (MI) continues to be a leading cause of mortality. Recent studies have suggested a novel cardioprotective role for allopurinol. This reviews aims to assess the efficacy of allopurinol in reducing the incidence of MI. Method: MEDLINE, Scopus, Web of Science, and Cochrane Library databases were searched for randomised controlled trials (RCT) examining the efficacy of allopurinol in reducing the incidence of MI. Additionally, reference lists of included studies were hand-searched. Data extraction and quality assessment were performed by two independent reviewers using tools endorsed by the Cochrane Collaboration. RevMan 5.3 was used to perform formal tests of heterogeneity, fixed-effects meta-analysis and leave-one-out sensitivity analyses. Results: 1034 citations were screened and only six studies satisfied the inclusion criterion. Published between 1988 and 1995, all of these studies examined the cardioprotective efficacy of allopurinol in the setting of coronary artery bypass graft (CABG) surgery. Perioperative MI was reported in $2(1.77 \%)$ of 113 patients randomised to allopurinol and $14(12.07 \%)$ of 116 control patients. A quality assessment revealed that methods of randomisation, allocation concealment and blinding were inconsistently reported. A fixed-effects meta-analysis $\left(\mathrm{I}^{2}=0 \%\right)$ identified a statistically significant reduced incidence of myocardial infarction (RR $0.21,95 \% \mathrm{Cl}: 0.06,0.70, \mathrm{p}$-value $=0.01$ ) in patients allocated to allopurinol. In leave-one-out sensitivity analyses, the 